International Journal of Pure and Applied Mathematics

Volume 91 No. 1 2014, 139-142

ISSN: 1311-8080 (printed version); ISSN: 1314-3395 (on-line version)

url: http://www.ijpam.eu

doi: http://dx.doi.org/10.12732/ijpam.v91i1.15

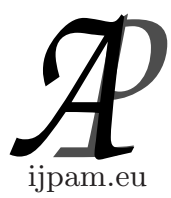

\title{
MORE ON THE DIOPHANTINE EQUATION $8^{x}+59^{y}=z^{2}$
}

\author{
Banyat Sroysang \\ Department of Mathematics and Statistics \\ Faculty of Science and Technology \\ Thammasat University, Rangsit Center \\ Pathumthani, 12121, THAILAND
}

\begin{abstract}
In this paper, we prove that $(1,0,3)$ is a unique non-negative integer solution $(x, y, z)$ for the Diophantine equation $8^{x}+59^{y}=z^{2}$ where $x, y$ and $z$ are non-negative integers.
\end{abstract}

AMS Subject Classification: 11D61

Key Words: exponential Diophantine equation

\section{Introduction}

In 2012, Sroysang [9] proved that $(1,0,3)$ is a unique non-negative integer solution $(x, y, z)$ for the Diophantine equation $8^{x}+19^{y}=z^{2}$ where $x, y$ and $z$ are non-negative integers.

In 2013, Rabago [6] showed that $(1,0,3),(1,1,5),(2,1,9)$ and $(3,1,23)$ are only four solutions $(x, y, z)$ for the Diophantine equation $8^{x}+17^{y}=z^{2}$ where $x, y$ and $z$ are non-negative integers.

In the same year, Sroysang [14] proved that the Diophantine equation $7^{x}+$ $8^{y}=z^{2}$ has no non-negative integer solution where $x, y$ and $z$ are non-negative integers. For related papers, we refer to $[1,2,3,4,7,8,10,11,12,13,15,16$, $17,18]$.

Received: January 14, 2014

(c) 2014 Academic Publications, Ltd. url: www.acadpubl.eu 
In this paper, we prove that $(1,0,3)$ is a unique solution $(x, y, z)$ for the Diophantine equation $8^{x}+59^{y}=z^{2}$ where $x, y$ and $z$ are non-negative integers.

\section{Preliminaries}

Proposition 2.1. [5] (Catalan's conjecture) $(3,2,2,3)$ is a unique solution $(a, b, x, y)$ for the Diophantine equation $a^{x}-b^{y}=1$ where $a, b, x$ and $y$ are integers such that $\min \{a, b, x, y\}>1$.

Lemma 2.2. [9] $(1,3)$ is a unique solution $(x, z)$ for the Diophantine equation $8^{x}+1=z^{2}$ where $x$ and $z$ are non-negative integers.

Lemma 2.3. The Diophantine equation $1+59^{y}=z^{2}$ has no non-negative integer solution.

Proof. Suppose that there are non-negative integers $y$ and $z$ such that $1+$ $59^{y}=z^{2}$. If $y=0$, then $z^{2}=2$ which is impossible. Thus, $y \geq 1$. Note that $z^{2}=1+59^{y} \geq 1+59^{1}=60$. Thus, $z \geq 8$. Now, we consider on the equation $z^{2}-59^{y}=1$. By Proposition 2.1, we obtain that $y=1$. Then $z^{2}=60$. This is a contradiction. Hence, the equation $1+59^{y}=z^{2}$ has no non-negative integer solution.

\section{Results}

Theorem 3.1. $(1,0,3)$ is a unique solution $(x, y, z)$ for the Diophantine equation $8^{x}+59^{y}=z^{2}$ where $x, y$ and $z$ are non-negative integers.

Proof. Let $x, y$ and $z$ be non-negative integers such that $8^{x}+59^{y}=z^{2}$. By Lemma 2.3, we obtain that $x \geq 1$. Then $z$ is odd. It follows that $z=2 t+1$ for some non-negative integer $t$. Then $8^{x}+59^{y}=4\left(t^{2}+t\right)+1$. It follows that $59^{y} \equiv 1(\bmod 4)$. Thus, $y$ is even. Then $y=2 k$ for some non-negative integer $k$. Now, we will divide the number $k$ into two cases.

Case $k=0$. Then $y=0$. By Lemma 2.2, we obtain that $x=1$ and $z=3$.

Case $k \geq 1$. . Note that $z^{2}-59^{2 k}=2^{3 x}$. It follows that $\left(z 5-59^{k}\right)\left(z+59^{k}\right)=$ $2^{3 x}$. Then $z-59^{k}=2^{u}$ and $z+59^{k}=2^{3 x-u}$ where $u$ is a non-negative integer. Then $2\left(59^{k}\right)=2^{3 x-u}-2^{u}=2^{u}\left(2^{3 x-2 u}-1\right)$. We have two subcases.

Subcase $u=0$. Then $z-59^{k}=1$. It follows that $z$ is even. This is a contradiction. 
Subcase $u=1$. We obtain that $2^{3 x-2}-1=59^{k}$. Then $2^{3 x-2}-59^{k}=1$. If $x=1$, then $k=0$ and then $y=0$. This implies that $x \geq 2$. By Proposition 2.1 , we have $k=1$. It follows that $2^{3 x-2}=60$. This is impossible.

Hence, $(1,0,3)$ is a unique solution $(x, y, z)$ for the equation $8^{x}+59^{y}=z^{2}$ where $x, y$ and $z$ are non-negative integers.

Corollary 3.2. The Diophantine equation $8^{x}+59^{y}=w^{4}$ has no nonnegative integer solution.

Proof. Suppose that there are non-negative integers $x, y$ and $w$ such that $8^{x}+59^{y}=w^{4}$. Let $z=w^{2}$. We obtain that $8^{x}+59^{y}=z^{2}$. By Theorem 3.1, we have $(x, y, z)=(1,0,3)$. It follows that $w^{2}=z=3$. This is a contradiction. Hence, the equation $8^{x}+59^{y}=w^{4}$ has no non-negative integer solution.

Corollary 3.3. $(3,0,3)$ is a solution $(u, y, z)$ for the Diophantine equation $2^{u}+59^{y}=z^{2}$.

Proof. This follows from Theorem 3.1 where $u=3 z$.

\section{References}

[1] D. Acu, On a Diophantine equation $2^{x}+5^{y}=z^{2}$, Gen. Math., 15 (2007), $145-148$.

[2] S. Chotchaisthit, On the Diophantine equation $2^{x}+11^{y}=z^{2}$, Maejo Int. J. Sci. Technol., 7 (2013), 291-293.

[3] S. Chotchaisthit, On the Diophantine equation $4^{x}+p^{y}=z^{2}$ where $p$ is a prime number, Amer. J. Math. Sci., 1 (2012), 191-193.

[4] S. Chotchaisthit, On the Diophantine equation $p^{x}+(p+1)^{y}=z^{2}$ where $p$ is a Mersenne prime, Int. J. Pure Appl. Math., 88 (2013), 169-172.

[5] P. Mihailescu, Primary cycolotomic units and a proof of Catalan's conjecture, J. Reine Angew. Math., 27 (2004), 167-195.

[6] J. F. T. Rabago, On an open problem by B. Sroysang, Konuralp J. Math., 1 (2013), 30-32.

[7] J. F. T. Rabago, On two Diophantine equations $3^{x}+19^{y}=z^{2}$ and $3^{x}+$ $91^{y}=z^{2}$, Int. J. Math. Sci. Comp., 3 (2013), 28-29. 
[8] B. Sroysang, More on the Diophantine equation $2^{x}+3^{y}=z^{2}$, Int. J. Pure Appl. Math., 84 (2013), 133-137.

[9] B. Sroysang, More on the Diophantine equation $8^{x}+19^{y}=z^{2}$, Int. J. Pure Appl. Math., 81 (2012), 601-604.

[10] B. Sroysang, On the Diophantine equation $3^{x}+5^{y}=z^{2}$, Int. J. Pure Appl. Math., 81 (2012), 605-608.

[11] B. Sroysang, On the Diophantine equation $3^{x}+17^{y}=z^{2}$, Int. J. Pure Appl. Math., 89 (2013), 111-114.

[12] B. Sroysang, On the Diophantine equation $5^{x}+7^{y}=z^{2}$, Int. J. Pure Appl. Math., 89 (2013), 115-118.

[13] B. Sroysang, On the Diophantine equation $5^{x}+23^{y}=z^{2}$, Int. J. Pure Appl. Math., 89 (2013), 119-122.

[14] B. Sroysang, On the Diophantine equation $7^{x}+8^{y}=z^{2}$, Int. J. Pure Appl. Math., 84 (2013), 111-114.

[15] B. Sroysang, On the Diophantine equation $23^{x}+32^{y}=z^{2}$, Int. J. Pure Appl. Math., 84 (2013), 231-234.

[16] B. Sroysang, On the Diophantine equation $31^{x}+32^{y}=z^{2}$, Int. J. Pure Appl. Math., 81 (2012), 609-612.

[17] B. Sroysang, On the Diophantine equation $47^{x}+49^{y}=z^{2}$, Int. J. Pure Appl. Math., 89 (2013), 279-282.

[18] B. Sroysang, On the Diophantine equation $89^{x}+91^{y}=z^{2}$, Int. J. Pure Appl. Math., 89 (2013), 283-286. 